\title{
Identification of protective antigens for vaccination against systemic salmonellosis
}

\section{Dirk Bumann*}

Focal Area Infection Biology, Biozentrum, University of Basel, Basel, Switzerland

\section{Edited by:}

Constantino López-Macías, Mexican Social Security Institute, Mexico;

University of Oxford, UK

\section{Reviewed by:}

Stephen McSorley, University of California Davis, USA

Emilio Luis Malchiodi, University of Buenos Aires, Argentina

Cristina Gil-Cruz, Cantonal Hospital

St. Gallen, Switzerland

*Correspondence:

Dirk Bumann, Focal Area Infection Biology, Biozentrum, University of Basel, CH-4056 Basel, Switzerland

e-mail:dirk.bumann@unibas.ch
There is an urgent medical need for improved vaccines with broad serovar coverage and high efficacy against systemic salmonellosis. Subunit vaccines offer excellent safety profiles but require identification of protective antigens, which remains a challenging task. Here, I review crucial properties of Salmonella antigens that might help to narrow down the number of potential candidates from more than 4000 proteins encoded in Salmonella genomes, to a more manageable number of 50-200 most promising antigens. I also discuss complementary approaches for antigen identification and potential limitations of current pre-clinical vaccine testing.

Keywords: Salmonella enterica, protective immunity, mouse model, human clinical trials, antigen expression, immunodominance, typhoid vaccines

\section{INTRODUCTION}

Salmonella enterica serovars Typhi and Paratyphi A, B, and C cause human enteric fever with an estimated annual number deaths of 190,000 (1). Enteric fever disease burden is probably underestimated because of difficult and insensitive diagnosis methods (2). In addition to these serovars, specific strains of serovar Typhimurium, which usually causes self-limiting gastroenteritis, can also cause systemic disease, particularly in young HIVinfected children in sub-Saharan Africa (invasive non-typhoidal salmonellosis, iNTS) (3).

Enteric fever and iNTS become increasingly difficult to treat with antibiotics because of rising resistance to fluoroquinolones and cephalosporins, and new drug candidates for these and other Gram-negative pathogens are scarce suggesting a risk of an increasing number of untreatable cases $(2,4)$.

\section{THE NEED FOR NOVEL SUBUNIT VACCINES}

Enteric fever can be prevented with a variety of vaccines (5). Killed whole-cell preparations of serovars Typhi and Paratyphi were successfully used to diminish incidence in endemic areas, but their use was discontinued because of frequent adverse reactions (6). A live attenuated $S$. Typhi strain Ty21a that was generated by chemical mutagenesis confers a moderate level of protection for up to three years against serovar Typhi, but not other relevant serovars (6). Additional genetically modified Salmonella strains have been tested in clinical trials with some success, but none of them has yet reached approval. Finally, the purified capsular carbohydrate Vi of serovar Typhi induces protective immunity over several years against serovars Typhi (6) and possibly Paratyphi C, but not Paratyphi A and B or Typhimurium that all lack such a capsule. Conjugation of Vi with an unrelated protein antigen improves immune response in small infants, a major target population for enteric fever (6). To cover the important serovar Paratyphi A, current efforts focus on linking the $\mathrm{O}$ antigen (carbohydrate part of lipopolysaccharide) with a protein antigen (7).

In conclusion, treatment of systemic salmonellosis becomes increasingly difficult, and prevention with currently available vaccines is hampered by only moderate levels and limited duration of protection, and incomplete coverage of clinically relevant serovars. This situation generates an urgent medical need for improved Salmonella vaccines.

Live attenuated Salmonella strains offer important advantages such as low production costs and oral administration, but pose a risk of causing disease especially in immunocompromised patients that might be inadvertently exposed, e.g., household contacts of vaccines that shed live Salmonella. Whole-cell killed vaccines are effective but contain pyogenic components that cause unacceptable inflammatory responses. As a consequence, development focuses on subunit vaccines that contain one or several key antigens inducing protective immune responses.

The key challenge of developing such a vaccine is identification of suitable antigens. Unfortunately, among thousands of potential Salmonella antigen candidates, probably only very few have the necessary properties. Efficient strategies to identify protective antigens among large number of candidates have been developed and applied for vaccines that protect against extracellular pathogens using inhibitory/bactericidal antibodies (reverse vaccinology) (8). For these pathogens, suitable antigens need to be surface exposed to enable antibody binding, which substantially narrows down the number of potential candidates. Furthermore, immunization trials can be scored for inhibitory/bactericidal antibodies using rather simple assays amenable for high-throughput. 
In contrast, similar strategies have not yet been developed for intracellular pathogens like Salmonella (which reside mostly in host macrophages during systemic disease), since criteria for preselecting promising antigens are unclear for most such pathogens, and immune correlates of protection remain poorly characterized. Antibodies (or just B cells) often contribute to protection but $\mathrm{T}$ cell responses are usually also required. The crucial $\alpha \beta \mathrm{T}$ cells recognize peptide epitopes and this led to a focus on protein antigens. Most intracellular pathogen genomes encode thousands of proteins, and identification of the few protective antigens among these numerous candidates remains challenging.

However, extensive recent work on Salmonella has uncovered some information that might be useful as a rational basis for future vaccine development against this and possibly other intracellular pathogens. In particular, coverage of relevant Salmonella strains, antigen expression in infected host tissues, and antigen compartmentalization within the Salmonella cell may substantially narrow down the number of promising antigen candidates.

\section{ANTIGENS ENABLING BROAD SEROVAR COVERAGE}

To achieve protective immunity against all relevant Salmonella strains, conserved antigens must be used. Hundreds of genes are missing or dysfunctional due to frameshift mutations or premature stop codons in certain relevant strains (9), but the rapidly increasing collection of genome sequences facilitates identification of suitable broadly conserved antigens. Orthologs usually share extensive sequence identity, but rare non-synonymous point mutations might still affect potentially crucial immunity determinants such as surface-exposed loops of outer membrane proteins (10). The 3D structures of many Salmonella proteins have been determined, and additional structures can be modeled based on homologs. However, it remains challenging to estimate which amino acid differences might impair cross-protective immune responses. As a consequence, antigens with highly conserved sequence among relevant serovars might be prioritized. On the other hand, antigens that play a potentially crucial role in pathogenesis of only a subset of serovars such as typhoid toxin (11) could still be an important contributor to vaccine combinations containing multiple antigens.

\section{ANTIGEN EXPRESSION IN HOST TISSUES}

To detect and kill Salmonella, the immune system must recognize antigens that Salmonella expresses in infected host tissues. For animal infection models, purification of genetically engineered fluorescent Salmonella cells from infected tissue homogenates using flow cytometry yields sufficient material for large-scale proteome analysis $(12,13)$. The results reveal expression of more than 1800 Salmonella antigens in mouse spleen. As a caveat, this analysis misses most secreted Salmonella proteins that are lost during purification. This is important, since at least one secreted protein can confer moderate protection (14). While escaping proteomics of purified Salmonella, highly expressed secreted proteins can be identified based on transcriptional in vivo data $(15,16)$.

Recent advances in proteomics enable even absolute quantification of copy numbers per Salmonella cell for most detected antigens (10). High expression levels might facilitate immune recognition $(10,15)$, but our systematic analysis did not support that protective antigens are generally highly expressed (10). This could reflect extensive host-pathogen coevolution modulating expression levels and immunogenicity of antigens. However, despite the poor predictive power of quantitative expression levels, antigen expression itself remains a crucial precondition for protective immune responses.

Salmonella proteomes in human tissues have not yet been investigated. However, experimental infections of human volunteers have been done in the past $(17,18)$, and a well-controlled protocol has recently been established (19). Purification by flow cytometry similar to the mouse studies would require infection with a genetically modified Salmonella strain, and type and required quantities of biopsy material would need to be determined.

Salmonella virulence has been extensively characterized in the mouse typhoid fever model. These studies have identified more than 270 Salmonella genes that contribute to pathogenesis. In almost all cases, this evidence indicates expression of the respective antigens at least at some stage of the infection. Virulence phenotypes in human beings are also available in a few cases from vaccine trials with live attenuated Salmonella strains (20-22). These scarce human data are largely consistent with observations for the corresponding Salmonella mutants in the mouse model, but systematic comparisons are currently impossible due to the lack of human data for most potential virulence factors.

Using another indirect approach, large-scale studies have identified antibodies that specifically recognize dozens of Salmonella antigens in sera of acutely infected and convalescent patients or experimentally infected mice, but not uninfected controls (23, 24). The presence of such antibodies is a clear indication that the respective Salmonella antigens are expressed at least at some stages of infection. Interestingly, there is a considerable overlap in immune signature of murine and human salmonellosis. On the other hand, comparison with direct ex vivo proteome analysis of Salmonella purified from infected mouse spleen reveals that serum antibodies recognize only a small minority of the more than 1800 in vivo expressed Salmonella antigens. It is possible, that Salmonella antigens that induce specific antibodies are particularly accessible for the host immune system, and thus represent most promising vaccine antigen candidates. However, direct comparison of antibody titers in convalescent mice with antigen protectivity in immunization/challenge studies shows that serum antibody levels have poor predictive power for identifying suitable vaccine antigens $(10,23)$. In fact, several of the most protective vaccine antigens failed to elicit detectable antibody responses in both mice and human beings, while immunodominant antigens mostly fail to protect.

Similar to antibody response in convalescent individuals, $\mathrm{T}$ cell responses to specific Salmonella antigens provide information about antigen expression during infection. CD4 T cell epitopes have been comprehensively predicted based on peptide properties that facilitate binding to antigen-presenting major histocompatibility complex (MHC) II molecules and T cell receptors (25). Some antigens were experimentally confirmed to be recognized by $\mathrm{T}$ cells from infected human beings $(26,27)$ and mice $(14,25,28,29)$, but not uninfected individuals. These results confirmed expression of corresponding Salmonella antigens (including the promising antigen SseB) at least during some stages of infection. Again, these 
identified antigens are only a small subset of all expressed antigens, and $\mathrm{T}$ cell responses during infection have poor predictive power for protective antigens (10). This might reflect expression at infection stages (29) or in distinct tissue microenvironments (30) that have limited relevance for protective immunity.

In conclusion, proteomics and virulence phenotypes provide large-scale information on Salmonella antigen expression in infected mice. Together, some 2000 different Salmonella antigens are expressed during infection in the mouse typhoid fever model, and might thus represent potential vaccine antigens. Evidence for human infections is much more fragmentary and largely restricted to serum antibody and $\mathrm{T}$ cell responses.

\section{ANTIGEN COMPARTIMENTALIZATION}

The localization of an antigen within the Salmonella cell may have a major impact on its protectivity. In particular, live intact Salmonella can only be detected by the host immune system through recognition of surface-exposed/released antigens, since internal Salmonella antigens are shielded by the cell envelope. On the other hand, dead Salmonella might release antigens regardless of their initial localization. In many infection foci, live and dead Salmonella reside in close proximity (30), and recognition of dead Salmonella alone might be sufficient for activation of bystander cells containing live Salmonella, resulting in effective clearance of both live and dead Salmonella. However, a subset of live Salmonella resides in tissue regions without any dead Salmonella $(10,30)$, and these would escape detection/clearance by immune responses directed exclusively against internal Salmonella antigens. This working model is supported by previously identified protective antigens $(15,31-$ 33) and our systematic comparison of Salmonella antigens from different compartments (10): all identified protective antigens are surface exposed. A recent study extended this finding to a secreted virulence effector protein (14), supporting the hypothesis that antigens must be accessible on live Salmonella to confer protective immunity.

Surface exposure/secretion might represent a powerful criterion to narrow down the number of potentially promising Salmonella vaccine antigens. Surface-exposed outer membrane proteins can be identified based on primary sequence properties and have been tabulated in databases $(34,35)$. Interestingly, outer membrane-associated lipoproteins can also confer protective immunity, even when they likely localize to the shielded periplasmic side of the outer membrane (10). Possibly, such lipoproteins are released in outer membrane vesicles that are degraded in host cell lysosomes thus exposing lipoproteins to the antigenpresentation platforms. Outer membrane-associated lipoproteins can again be identified based on primary sequences (36). Experimental analysis of outer membrane preparations $(37,38)$ and/or biotinylated surface-exposed proteins (39) can be used to confirm theoretical predictions, and to identify additional exposed antigens that might be secreted through unconventional mechanisms.

In addition to surface-associated proteins, Salmonella translocates various proteins directly to the infected host cell cytosol, predominantly using the SPI-2 associated type III secretion system. SPI-2 effector proteins are intensively studied and the currently identified list of 32 proteins (40) might approximate completion. The SPI-2 translocon subunit SseB itself is one of the most promising vaccine antigens $(15,23,27)$. During initial phases of infection, Salmonella secretes proteins also through the SPI-1 associated type III secretion system and through the flagellar apparatus (in particular, flagellin, a moderately protective antigen) $(29,31)$.

Together, surface-exposed and secreted Salmonella antigens comprise some 200 different antigens, and at least around 50 of them are expressed during infection in the mouse typhoid model based on transcriptional data, proteomics, virulence phenotypes, and/or immunization data. Twenty-six such antigens have already been tested and nine appear to confer some degree of protective immunity in mouse typhoid fever immunization/challenge studies (FliC, SseB, OmpD, CirA, IroN, T0937, SlyB, PagN, and SseI; in some cases group sizes were too small to obtain definitive proof) $(10,14,15,29,31-33)$.

\section{FUTURE PERSPECTIVES}

Immunization/challenge experiments in the mouse typhoid fever model have shown that live attenuated Salmonella strains can provide full long-term protection against otherwise lethal challenge infections (41). Compared to this benchmark, progress with subunit vaccines in the same model has been somewhat disappointing. Despite large-scale experimental and computational screening campaigns in several different laboratories, few Salmonella antigens with at most moderate protectivity in the mouse typhoid fever model have been identified. None of these antigens confer full protection for more than some 30 days after challenge infection. This could reflect immune evasion of the challenge Salmonella strain by mutation of crucial epitopes within the respective antigens. During such a long infection time, other adaptive immune responses might be expected, but these responses are obviously insufficient for protective immunity.

It is possible that the best protective antigens have not yet been identified, or that multiple antigens need to be combined for full protection and prevention of immune evasion. It is also possible that antigens other than proteins, such as lipids, carbohydrates, or even small molecules (42), are necessary for high levels of protection. One approach to test this hypothesis could use progressive depletion of specific antigens from protective live attenuated Salmonella strains, by deleting respective biosynthesis genes. However, this approach is limited to non-essential genes and is thus non-informative for antigens such as riboflavin intermediates (12, 42). Alternatively, killed whole-cell vaccines might be fractionated and tested for protection. Unfortunately, killed whole-cell vaccine formulations with high protective efficacy in the mouse typhoid fever model have not yet been described. Future studies might revisit this issue, especially since killed whole-cell vaccines confer substantial protective immunity against invasive salmonellosis in human beings (although they are no longer used because of severe adverse reactions) (1).

Finally, it is important to consider what level of protection is actually needed in pre-clinical mouse models before proceeding to human clinical vaccine trials. In the typhoid fever model, genetically high-susceptible mouse strains defective for the divalent cation transporter Slc11a1 (NRAMP1) (43), are infected with doses of $S$. enterica serovar Typhimurium that result in an attack rate of $100 \%$. This combination reproduces some important aspects of human disease including Salmonella dissemination from intestinal 
sites, histopathology in spleen and liver (splenomegaly, formation of structured inflammatory lesions), relevance of various Salmonella virulence factors and host cytokines, and protective immunity against reinfection in convalescent individuals, or individuals vaccinated with live attenuated Salmonella strains (41). On the other hand, disease progression in mice is more rapid and always lethal when using wild-type Salmonella strains, in contrast to human enteric fever. Importantly, protective immunity against challenge infections in the mouse model requires both B cell and CD4 T cell responses (28), but neither antibodies (44) nor MHC I-restricted CD8 T cells (45). In contrast, vaccination-induced antibodies alone seem to confer already a substantial level of protection in human beings (46), at least in endemic areas where pre-existing immune responses to Salmonella are highly prevalent (24). It is thus possible that full protection against virulent wild-type Salmonella strains in genetically susceptible mice might be too stringent a criterion to judge vaccine efficacy.

Instead, it might be worth considering genetically resistant mice (47), in which antibodies seem to suffice for protective immunity (48), and heat-killed Salmonella mediate substantial immunity (49) similar to the situation in human beings (6). Interestingly, flagellin is also highly protective in resistant mice (50), in contrast to only moderate protectivity in susceptible mice.

In addition to the mouse strain, the challenge infection dose should be re-considered. Controlled human trials have shown that vaccine-induced immunity can be easily overwhelmed by even moderate challenge doses (17). Vaccines that have welldocumented efficacy in field-trials, completely fail to protect against $S$. Typhi when given at doses in the range of $10^{6}-10^{7} \mathrm{CFU}$. Vaccine efficacy is only seen at a much lower dose of $10^{5} \mathrm{CFU}$ that caused disease in only $40 \%$ of unvaccinated control volunteers [a recent study showed higher attack rates at such doses (19)]. Based on these human data, commonly used mouse challenge infections that result in $100 \%$ attack rates might be too stringent for revealing a moderate level of protective immunity that could still be sufficient for preventing even a large proportion of human disease under relevant field conditions.

Finally, a better understanding of human immune responses that are relevant for protective immunity could help to replace the crude readout parameter "survival after challenge infection" with more informative quantitative immune parameters. Ongoing studies in an experimental human infection and vaccination model (19) will likely provide such crucial information in the near future.

\section{CONCLUSION}

Several Salmonella antigens that can mediate at least partial protective immunity against lethal challenge infections in mice have recently been identified. Analysis of their properties suggests that efforts to identify further suitable antigens might focus on a limited number of promising surface-associated/secreted candidates that are expressed in infected host tissues. However, none of the known individual antigens mediates solid strong protection, comparable to what can be achieved with attenuated live Salmonella strains. Future studies could explore antigen combinations and possibly antigens other than proteins. Moreover, a better understanding of qualitative and quantitative immune parameters that are required to protect human beings is needed to guide pre-clinical models for further vaccine optimization and to determine what levels of protection are needed.

\section{ACKNOWLEDGMENTS}

I would like to thank past and present lab members for their contributions, and Deutsche Forschungsgemeinschaft and Schweizerischer Nationalfonds for funding.

\section{REFERENCES}

1. Lozano R, Naghavi M, Foreman K, Lim S, Shibuya K, Aboyans V, et al. Global and regional mortality from 235 causes of death for 20 age groups in 1990 and 2010: a systematic analysis for the Global Burden of Disease Study 2010. Lancet (2012) 380:2095-128. doi:10.1016/S0140-6736(12)61728-0

2. Crump JA, Mintz ED. Global trends in typhoid and paratyphoid fever. Clin Infect Dis (2010) 50:241-6. doi:10.1086/649541

3. Maclennan CA, Levine MM. Invasive nontyphoidal Salmonella disease in Africa: current status. Expert Rev Anti Infect Ther (2013) 11:443-6. doi:10.1586/ eri.13.27

4. Jean SS, Hsueh PR. High burden of antimicrobial resistance in Asia. Int J Antimicrob Agents (2011) 37:291-5. doi:10.1016/j.ijantimicag.2011.01.009

5. Martin LB. Vaccines for typhoid fever and other salmonelloses. Curr Opin Infect Dis (2012) 25:489-99. doi:10.1097/QCO.0b013e328356ffeb

6. Anwar E, Goldberg E, Fraser A, Acosta CJ, Paul M, Leibovici L. Vaccines for preventing typhoid fever. Cochrane Database Syst Rev (2014) 1:CD001261. doi:10.1002/14651858.CD001261.pub3

7. Maclennan CA, Martin LB, Micoli F. Vaccines against invasive Salmonella disease: current status and future directions. Hum Vaccin Immunother (2014) 10:33-48. doi:10.4161/hv.29054

8. Sette A, Rappuoli R. Reverse vaccinology: developing vaccines in the era of genomics. Immunity (2010) 33:530-41. doi:10.1016/j.immuni.2010.09.017

9. Nuccio SP, Baumler AJ. Comparative analysis of Salmonella genomes identifies a metabolic network for escalating growth in the inflamed gut. MBio (2014) 5:e929-914. doi:10.1128/mBio.00929-14

10. Barat S, Willer Y, Rizos K, Claudi B, Maze A, Schemmer AK, et al. Immunity to intracellular salmonella depends on surface-associated antigens. PLoS Pathog (2012) 8:e1002966. doi:10.1371/journal.ppat.1002966

11. Song J, Gao X, Galan JE. Structure and function of the Salmonella Typhi chimaeric A(2)B(5) typhoid toxin. Nature (2013) 499:350-4. doi:10.1038/ nature 12377

12. Becker D, Selbach M, Rollenhagen C, Ballmaier M, Meyer TF, Mann M, et al. Robust Salmonella metabolism limits possibilities for new antimicrobials. Nature (2006) 440:303-7. doi:10.1038/nature04616

13. Steeb B, Claudi B, Burton NA, Tienz P, Schmidt A, Farhan H, et al. Parallel exploitation of diverse host nutrients enhances Salmonella virulence. PLoS Pathog (2013) 9:e1003301. doi:10.1371/journal.ppat.1003301

14. Kurtz JR, Petersen HE, Frederick DR, Morici LA, Mclachlan JB. Vaccination with a single CD4 T cell peptide epitope from a Salmonella type III-secreted effector protein provides protection against lethal infection. Infect Immun (2014) 82:2424-33. doi:10.1128/IAI.00052-14

15. Rollenhagen C, Sorensen M, Rizos K, Hurvitz R, Bumann D. Antigen selection based on expression levels during infection facilitates vaccine development for an intracellular pathogen. Proc Natl Acad Sci U S A (2004) 101:8739-44. doi:10.1073/pnas.0401283101

16. Rollenhagen C, Bumann D. Salmonella enterica highly expressed genes are disease specific. Infect Immun (2006) 74:1649-60. doi:10.1128/IAI.74.3.1649-1660. 2006

17. Woodward WE. Volunteer studies of typhoid fever and vaccines. Trans $R$ Soc Trop Med Hyg (1980) 74:553-6. doi:10.1016/0035-9203(80)90133-9

18. Levine MM, Tacket CO, Sztein MB. Host-Salmonella interaction: human trials. Microbes Infect (2001) 3:1271-9. doi:10.1016/S1286-4579(01)01487-3

19. Waddington CS, Darton TC, Jones C, Haworth K, Peters A, John T, et al. An outpatient, ambulant-design, controlled human infection model using escalating doses of Salmonella Typhi challenge delivered in sodium bicarbonate solution. Clin Infect Dis (2014) 58:1230-40. doi:10.1093/cid/ciu078 
20. Bumann D, Hueck C, Aebischer T, Meyer TF. Recombinant live Salmonella spp. for human vaccination against heterologous pathogens. FEMS Immunol Med Microbiol (2000) 27:357-64. doi:10.1111/j.1574-695X.2000.tb01450.x

21. Tacket CO, Levine MM. CVD 908, CVD 908-htrA, and CVD 909 live oral typhoid vaccines: a logical progression. Clin Infect Dis (2007) 45(Suppl 1):S20-3. doi: $10.1086 / 518135$

22. Frey SE, Lottenbach KR, Hill H, Blevins TP, Yu Y, Zhang Y, et al. A Phase I, doseescalation trial in adults of three recombinant attenuated Salmonella Typhi vaccine vectors producing Streptococcus pneumoniae surface protein antigen PspA. Vaccine (2013) 31:4874-80. doi:10.1016/j.vaccine.2013.07.049

23. Lee SJ, Liang L, Juarez S, Nanton MR, Gondwe EN, Msefula CL, et al. Identification of a common immune signature in murine and human systemic Salmonellosis. Proc Natl Acad Sci U S A (2012) 109:4998-5003. doi:10.1073/pnas. 1111413109

24. Liang L, Juarez S, Nga TV, Dunstan S, Nakajima-Sasaki R, Davies DH, et al. Immune profiling with a Salmonella Typhi antigen microarray identifies new diagnostic biomarkers of human typhoid. Sci Rep (2013) 3:1043. doi:10.1038/ srep01043

25. Maybeno M, Redeker A, Welten SP, Peters B, Loughhead SM, Schoenberger SP, et al. Polyfunctional CD4(+) T cell responses to immunodominant epitopes correlate with disease activity of virulent Salmonella. PLoS One (2012) 7:e43481. doi:10.1371/journal.pone.0043481

26. Sheikh A, Khanam F, Sayeed MA, Rahman T, Pacek M, Hu Y, et al. Interferongamma and proliferation responses to Salmonella enterica serotype Typhi proteins in patients with S. Typhi Bacteremia in Dhaka, Bangladesh. PLoS Negl Trop Dis (2011) 5:e1193. doi:10.1371/journal.pntd.0001193

27. Reynolds CJ, Jones C, Blohmke CJ, Darton TC, Goudet A, Sergeant R, et al. The serodominant secreted effector protein of Salmonella, SseB, is a strong CD4 antigen containing an immunodominant epitope presented by diverse HLA class II alleles. Immunology (2014):doi:10.1111/imm.12327

28. Cookson BT, Bevan MJ. Identification of a natural T cell epitope presented by Salmonella-infected macrophages and recognized by $\mathrm{T}$ cells from orally immunized mice. J Immunol (1997) 158:4310-9.

29. Lee SJ, Mclachlan JB, Kurtz JR, Fan D, Winter SE, Baumler AJ, et al. Temporal expression of bacterial proteins instructs host CD4 $\mathrm{T}$ cell expansion and th17 development. PLoS Pathog (2012) 8:e1002499. doi:10.1371/journal.ppat. 1002499

30. Burton NA, Schurmann N, Casse O, Steeb AK, Claudi B, Zankl J, et al. Disparate impact of oxidative host defenses determines the fate of Salmonella during systemic infection in mice. Cell Host Microbe (2014) 15:72-83. doi:10.1016/j.chom.2013.12.006

31. McSorley SJ, Cookson BT, Jenkins MK. Characterization of CD4+ T cell responses during natural infection with Salmonella Typhimurium. J Immunol (2000) 164:986-93. doi:10.4049/jimmunol.164.2.986

32. Gil-Cruz C, Bobat S, Marshall JL, Kingsley RA, Ross EA, Henderson IR, et al. The porin OmpD from nontyphoidal Salmonella is a key target for a protective B1b cell antibody response. Proc Natl Acad Sci U S A (2009) 106:9803-8. doi:10.1073/pnas.0812431106

33. Yang Y, Wan C, Xu H, Aguilar ZP, Tan Q, Xu F, et al. Identification of an outer membrane protein of Salmonella enterica serovar Typhimurium as a potential vaccine candidate for Salmonellosis in mice. Microbes Infect (2013) 15:388-98. doi:10.1016/j.micinf.2013.02.005

34. Paramasivam N, Linke D. ClubSub-P: cluster-based subcellular localization prediction for Gram-negative bacteria and archaea. Front Microbiol (2011) 2:218. doi:10.3389/fmicb.2011.00218

35. Freeman TC Jr., Wimley WC. TMBB-DB: a transmembrane beta-barrel proteome database. Bioinformatics (2012) 28:2425-30. doi:10.1093/bioinformatics/ bts478

36. Diaz-Mejia JJ, Babu M, Emili A. Computational and experimental approaches to chart the Escherichia coli cell-envelope-associated proteome and interactome. FEMS Microbiol Rev (2009) 33:66-97. doi:10.1111/j.1574-6976.2008.00141.x

37. Chooneea D, Karlsson R, Encheva V, Arnold C, Appleton H, Shah H. Elucidation of the outer membrane proteome of Salmonella enterica serovar Typhimurium utilising a lipid-based protein immobilization technique. BMC Microbiol (2010) 10:44 doi:10.1186/1471-2180-10-44

38. Brown RN, Sanford JA, Park JH, Deatherage BL, Champion BL, Smith RD, et al. A comprehensive subcellular proteomic survey of Salmonella grown under phagosome-mimicking versus standard laboratory conditions. Int J Proteomics (2012) 2012:123076. doi:10.1155/2012/123076

39. Sabarth N, Lamer S, Zimny-Arndt U, Jungblut PR, Meyer TF, Bumann D. Identification of surface-exposed proteins of Helicobacter pylori by selective biotinylation, affinity purification, and two-dimensional gel electrophoresis. J Biol Chem (2002) 277:27896-902. doi:10.1074/jbc.M204473200

40. Figueira R, Watson KG, Holden DW, Helaine S. Identification of Salmonella pathogenicity island-2 type III secretion system effectors involved in intramacrophage replication of $S$. enterica serovar typhimurium: implications for rational vaccine design. MBio (2013) 4:e00065. doi:10.1128/mBio.00065-13

41. Dougan G, John V, Palmer S, Mastroeni P. Immunity to salmonellosis. Immunol Rev (2011) 240:196-210. doi:10.1111/j.1600-065X.2010.00999.x

42. Corbett AJ, Eckle SB, Birkinshaw RW, Liu L, Patel O, Mahony J, et al. T-cell activation by transitory neo-antigens derived from distinct microbial pathways. Nature (2014) 509:361-5. doi:10.1038/nature13160

43. Valdez Y, Ferreira RB, Finlay BB. Molecular mechanisms of Salmonella virulence and host resistance. Curr Top Microbiol Immunol (2009) 337:93-127. doi:10.1007/978-3-642-01846-6_4

44. Nanton MR, Way SS, Shlomchik MJ, McSorley SJ. Cutting edge: B cells are essential for protective immunity against Salmonella independent of antibody secretion. J Immunol (2012) 189:5503-7. doi:10.4049/jimmunol.1201413

45. Lee SJ, Dunmire S, McSorley SJ. MHC class-I-restricted CD8 T cells play a protective role during primary Salmonella infection. Immunol Lett (2012) 148:138-43. doi:10.1016/j.imlet.2012.10.009

46. Strugnell RA, Scott TA, Wang N, Yang C, Peres N, Bedoui S, et al. Salmonella vaccines: lessons from the mouse model or bad teaching? Curr Opin Microbiol (2014) 17:99-105. doi:10.1016/j.mib.2013.12.004

47. Simon R, Tennant SM, Galen JE, Levine MM. Mouse models to assess the efficacy of non-typhoidal Salmonella vaccines: revisiting the role of host innate susceptibility and routes of challenge. Vaccine (2011) 29:5094-106. doi:10.1016/j.vaccine.2011.05.022

48. Eisenstein TK, Angerman CR. Immunity to experimental Salmonella infection: studies on the protective capacity and immunogenicity of lipopolysaccharide, acetone-killed cells, and ribosome-rich extracts of Salmonella typhimurium in C3H/HeJ and CD-1 mice. J Immunol (1978) 121:1010-4.

49. Herzberg M, Nash P, Hino S. Degree of immunity induced by killed vaccines to experimental salmonellosis in mice. Infect Immun (1972) 5:83-90.

50. Simon R, Tennant SM, Wang JY, Schmidlein PJ, Lees A, Ernst RK, et al. Salmonella enterica serovar enteritidis core $\mathrm{O}$ polysaccharide conjugated to $\mathrm{H}: \mathrm{g}, \mathrm{m}$ flagellin as a candidate vaccine for protection against invasive infection with $\mathrm{S}$. enteritidis. Infect Immun (2011) 79:4240-9. doi:10.1128/IAI.05484-11

Conflict of Interest Statement: The author declares that the research was conducted in the absence of any commercial or financial relationships that could be construed as a potential conflict of interest.

Received: 18 June 2014; paper pending published: 15 July 2014; accepted: 25 July 2014; published online: 11 August 2014.

Citation: Bumann D (2014) Identification of protective antigens for vaccination against systemic salmonellosis. Front. Immunol. 5:381. doi: 10.3389/fimmu.2014.00381

This article was submitted to Microbial Immunology, a section of the journal Frontiers in Immunology.

Copyright (c) 2014 Bumann. This is an open-access article distributed under the terms of the Creative Commons Attribution License (CC BY). The use, distribution or reproduction in other forums is permitted, provided the original author(s) or licensor are credited and that the original publication in this journal is cited, in accordance with accepted academic practice. No use, distribution or reproduction is permitted which does not comply with these terms. 